\title{
An Automated Age-Related Macular Degeneration Classification Based on Local Texture Features in Optical Coherence Tomography Angiography
}

DOI:

10.1007/978-3-319-95921-4_19

\section{Document Version}

Accepted author manuscript

Link to publication record in Manchester Research Explorer

Citation for published version (APA):

Alfahaid, A., \& Morris, T. (2018). An Automated Age-Related Macular Degeneration Classification Based on Local Texture Features in Optical Coherence Tomography Angiography. In M. Nixon, S. Mahmoodi, \& R. Zwiggelaar (Eds.), Medical Image Understanding and Analysis - 22nd Conference, Proceedings (pp. 189-200).

(Communications in Computer and Information Science; Vol. 894). https://doi.org/10.1007/978-3-319-95921-4_19

\section{Published in:}

Medical Image Understanding and Analysis - 22nd Conference, Proceedings

\section{Citing this paper}

Please note that where the full-text provided on Manchester Research Explorer is the Author Accepted Manuscript or Proof version this may differ from the final Published version. If citing, it is advised that you check and use the publisher's definitive version.

\section{General rights}

Copyright and moral rights for the publications made accessible in the Research Explorer are retained by the authors and/or other copyright owners and it is a condition of accessing publications that users recognise and abide by the legal requirements associated with these rights.

\section{Takedown policy}

If you believe that this document breaches copyright please refer to the University of Manchester's Takedown Procedures [http://man.ac.uk/04Y6Bo] or contact uml.scholarlycommunications@manchester.ac.uk providing relevant details, so we can investigate your claim.

\section{OPEN ACCESS}




\title{
An automated age-related macular degeneration classification based on local texture features in optical coherence tomography angiography
}

\author{
Abdullah Alfahaid ${ }^{1,2}$, Tim Morris ${ }^{1}$ \\ ${ }^{1}$ School of Computer Science, University of Manchester, Oxford Road, Manchester, M13 9PL \\ ${ }^{2}$ College of Computer Science and Engineering at Yanbu, Taibah University, Medina, KSA
}

\begin{abstract}
In this paper, an age-related macular degeneration (AMD) classification algorithm based on local texture features is proposed to support the automated analysis of optical coherence tomography angiography (OCTA) images in wet AMD. The algorithm is based on rotation invariant uniform Local $B$ inary Patterns $\left(L B P S^{r i u 2}\right)$ as a texture measurement technique. It was chosen due to its computational simplicity and its invariance against any transformation of the grey level as well as against texture orientation change. The texture features are extracted from the whole image without targeting a particular area. The algorithm was tested on two-dimensional angiogram greyscale images of four different retinal layers acquired via OCTA scan. The evaluation was performed using a ten-fold cross-validation strategy applied to a set of 184 OCTA images consisting of 92 normal control and 92 wet AMD images. The classification was performed on each separate retinal layer, and on all layers together. According to the results, the algorithm was able to achieve a promising performance with mean accuracy of $89 \%$ for all layers together and $89 \%, 94 \%, 98 \%$ and $100 \%$ for the superficial, deep, outer and choriocapillaris layers respectively.
\end{abstract}

Keywords: wet AMD, local texture features, OCTA, $L B P s^{\text {riu } 2}$

\section{Introduction}

Age-related macular degeneration (AMD) is a painless eye condition commonly found in people aged over 50 and is the leading cause of vision loss in this age group. This condition affects the macula (the central region of the retina) leading to central vision loss. The late stage of AMD, known as wet AMD, is characterised by the formation of new blood vessels under the macula. Regrettably, the early stages of wet AMD are not observable by patients and can result in significant damage to the retina leading to blindness. Early detection and diagnosis of this disease are essential for the purpose of offering treatment options to prevent or reduce the possibility of vision loss [1].

Various eye imaging techniques such as optical coherence tomography (OCT) have been developed to assist ophthalmologists in detecting and examining eye diseases 
that affect the retinal vasculature, such as wet AMD. The clear visualisation of retinal vasculature constitutes a critical element in the detection of wet AMD, however, OCT is limited in this regard [2]. An advanced technique of the OCT scan known as optical coherence tomography angiography (OCTA) has recently been developed. This utilises motion contrast scanning of the flow of volumetric blood information producing clear angiographic images in a fraction of a second [2], [3]. Advantageous methods such as dye-free angiography and non-invasive three-dimensional imaging are also supported by OCTA. Since OCTA scans produce images for the various blood vessels of the choroid and retinal layers, changes in the blood vessels can be detected, making it a particularly suitable technique for identifying choroidal neovascularisation (CNV) in wet AMD [3].

The patterns of retinal vasculature structure, in particular retinal layers, are affected by wet AMD. These layers are the superficial inner retina, deep inner retina, outer retina and choriocapillaris. Ease of distinguishing blood vessel abnormalities varies between these layers, presenting less of an effect in certain layers than in others. At times abnormalities cannot be detected visually. Figure 1 shows four different retinal layers images captured using an OCTA scan for an eye of a patient with wet AMD condition and for a healthy eye.

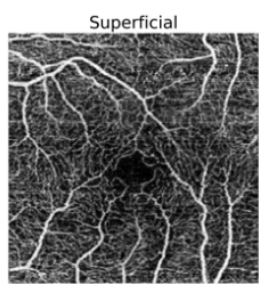

Superficial

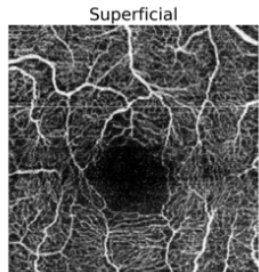

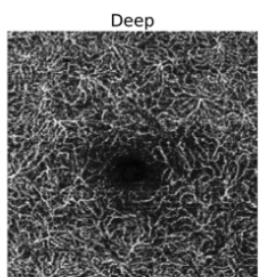

Deep

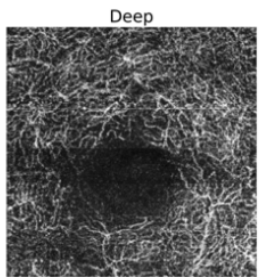

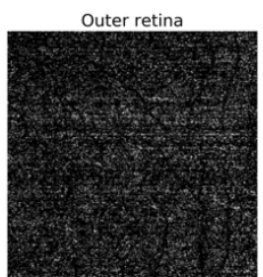

Outer retina

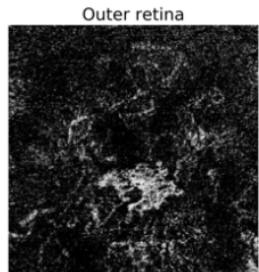

Choriocapillaris

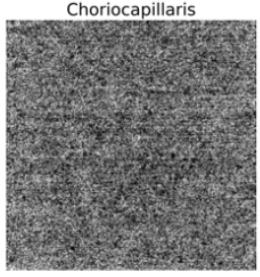

Choriocapillaris

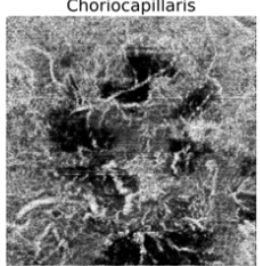

Fig. 1. The first row shows four different retinal layers (superficial retina, deep inner retina, outer retina and choriocapillaris) of a healthy eye and the second row shows the same retinal layers for the eye of a patient with wet AMD. It can be seen that the abnormalities in the blood vessel patterns vary between the different layers; they are clearer in the outer and choriocapillaris layers as compared to the other layers.

The accurate detection and quantification of the CNV form in wet AMD patients would be particularly useful to ophthalmologists in their evaluation and diagnosis process, the consequences of which would determine the various treatments to be used [4]. As observed from the previous figure, the texture of OCTA images is affected by wet AMD. Ophthalmologists currently rely on the textural appearance of these images in their diagnostic process [1]. The simplest technique currently used for 
quantifying $\mathrm{CNV}$ form in wet $\mathrm{AMD}$ is that of manually tracing the border of the abnormal blood vessel regions [4]. However, this technique is time-consuming and subjective as it involves manual segmentation of the regions of interest and therefore its clinical utility is reduced. Moreover, evidence shows that recognising textural information relevant to the spectral properties or higher-order statistics (HOS) of an image is problematic for the human eye [5].

Automated methods that provide extraction of discriminative textural features are desirable as they could assist ophthalmologists in extracting the features that relate to a specific eye disease but are difficult to distinguish visually. Additionally, they could improve the diagnostic process by reducing the ophthalmologist's workload. In this paper, a novel classification algorithm is presented for the detection of wet AMD in OCTA images. The proposed approach uses texture features based on the rotation invariant $u$ niform Local Binary Patterns ( $L B P s^{r i u 2}$ ) descriptor introduced in [6].

\section{Related work}

There is very little published work on the analysis of OCTA images for patients with wet AMD. One study [1], however, used OCTA images to measure the CNV area with a manual assistance for the purpose of identifying wet AMD features. A method was developed in [7] with a view to automating the analysis of OCTA images to detect the CNV area in wet AMD. A recent study [4] proposed an automated method based on the segmentation of $\mathrm{CNV}$ area at the outer retina layer to enable the detection of wet AMD using OCTA images. Algorithms in both [4] and [7] involve a preprocessing step for OCTA images to reduce noise areas by applying the Gaussian filter to obtain a clear image that highlights the $\mathrm{CNV}$ region. While this step is helpful in reducing noise, and enables the CNV region to be clearly visible, it excludes some of the CNV regions [4], [7]. However, it is believed that change in retinal vasculature structure is one of the key indicators of CNV in wet AMD and hence their clinical application is reduced since these methods are prone to error in diagnosis.

Previously mentioned methods are based on segmentation of retinal vascular areas. Based on the segmented areas, some measurements are derived to identify the features related to normal and diseased retinas. However, it was observed that deriving accurate measurements is difficult with these methods. This can be due to the complex characteristics of the diseases, noise caused by patients' motion while capturing the images and can be influenced by human subjectivity when manual grading is involved. It is true that a number of techniques can be used to reduce or remove noise from images such as the Gaussian filter used in [4] and [7]. However, when applying this filter, image details may change as occurred in [4] and [7] when some of the CNV regions were excluded. To the best of our knowledge, other techniques based on texture analysis using OCTA images and performing wet AMD classification have not been investigated yet. Therefore, this study focuses on improving upon the previously stated method and creates an automated wet AMD classification algorithm based on local texture features. 


\section{Proposed approach}

To overcome the various limitations of previous methods, the proposed algorithm aims to avoid employing any pre-processing steps (smoothing or filtering images) as it may change image details, as occurred in [4] and [7]. It also precludes the need for manual assistance. It uses the raw data (OCTA images) and is completely automated. The approach simply consists of two main steps. First, feature extraction, whereby the $L B P s^{\text {riu } 2}$ features are extracted from all images (constructing the feature vectors); second, classification, using the features obtained (passing the feature vectors onto a classifier to classify subsequent yet unseen OCTA images). Segmentation is unnecessary since the algorithm combines both statistical approaches (computes the histograms) and structural approaches (detects the micro-structures) on image texture patterns. As a result, the proposed method would be particularly useful for wet AMD, even when its characteristics are complicated, and this is not the case with segmentation-based techniques, since deriving accurate measurements is too difficult to achieve.

\subsection{Feature extraction}

The Local Binary Patterns $(L B P)$ is a texture descriptor that first appeared in [8] and became popular by the introduction of the $L B P s^{r i u 2}$ descriptor in [6]. The LBPs ${ }^{r i u 2}$ was chosen for use with this algorithm due to its outstanding advantages over many existing texture descriptors, as follows [6]:

1. Invariance against illumination intensity changes, a common issue with OCTA images [9].

2. Invariance against rotation changes (ensuring that all images captured have a similar rotation can be challenging).

3. Discriminative power of the features produced.

4. No need to tune complex parameters.

5. Low computational complexity.

6. Ease of implementation.

The $L B P s^{\text {riu } 2}$ removes sensitivity to the rotation of texture images by computing the patterns within a symmetrical circular neighbourhood bitwise right shift and considers only the patterns that are rotationally uniform, hence it is rotation $i$ nvariant [6]. It is also invariant against any monotonic transformation of the grey level change since it considers the value of subtracting a grey value of a central pixel from a grey value of local neighbourhood pixel rather than the pixels' inherent value [6]. The binary pattern in the $L B P s^{\text {riu }}$ descriptor is simply formed by thresholding a grey value of local neighbourhood pixel according to a grey value of a central pixel and then it is measured according to the given operator:

$L B P s_{\underline{p}, \underline{r}}^{r i u 2}=\left\{\begin{array}{cc}\sum_{\underline{p}=0}^{\underline{p}-1} s\left(g_{p}-g_{\underline{c}}\right) \text { if } u\left(L B P s_{\underline{p}, \underline{r}}\right) \leq 2 \\ \underline{p}+1 & \text { otherwise }\end{array}, \quad s(x)= \begin{cases}1, & x \geq 0 \\ 0, & x<0\end{cases}\right.$ 
Where

$u\left(L B P s_{\underline{p}, \underline{\underline{r}}}\right)=\left|s\left(g_{\underline{p}-1}-g_{\underline{c}}\right)-s\left(g_{0}-g_{\underline{c}}\right)\right|+\sum_{p=1}^{\underline{p}-1}\left|s\left(g_{p}-g_{\underline{c}}\right)-s\left(g_{p-1}-g_{\underline{c}}\right)\right|$

Where $u\left(L B P s_{p, \underline{r}}\right)$ is the uniformity measure technique that counts the number of bitwise $(0 / 1$ or $1 / 0)$ transitions that appear in the binary pattern and the maximum number of transitions for each pattern to be considered as a uniform is two; otherwise it is non- $u$ niform. According to [6] the superscript ( ${ }^{r i u 2}$ ) indicates using the Local $B$ inary $P$ atterns that have at most two transitions " $u$ niform" and also rotation invariant. $g_{\underline{c}}$ is the grey value of central pixel $(\underline{\mathcal{c}})$ and $g_{p}$ is the grey value of local neighbourhood pixel where $p=(0,1, \ldots, p-1) . p$ is the number of local neighbourhood pixels that equally spread out around a circle of radius $\underline{r}$, where $\underline{r}>0$ and $\underline{p}>1$. When the coordinate of $\left(g_{\underline{c}}\right)$ is $(0,0)$, the coordinates of all $g_{p}$ are calculated by $(-\underline{r}$ $\sin (\pi p / \underline{p}), \underline{r} \cos (\pi p / \underline{p}))$. If any of the $g_{p}$ does not fall accurately within the centre of an image pixel, its location is then estimated by interpolation.

The number of $u$ niform binary patterns that can occur in the $L B P s^{\text {riu } 2}$ with a symmetrical circular set of pixels $\underline{p}$ is $(\underline{p}+1)$. To precisely measure the texture of OCTA images, there are two parameters with the LBPS $s_{p, \underline{r}}^{r i u 2}$ that have to be set properly. The first parameter is $p$, which defines the number of local neighbourhood pixels as well as the dimensionality of the histogram. The second parameter is $\underline{r}$, which defines the distance between the local neighbourhood pixels and the central pixel. The $L B P s^{r i u 2}$ patterns were extracted from each image and then each image was described by a histogram with $(p+2)$ bins dimensional that counts the number of times of the $L B P s^{r i u 2}$ patterns occur within each image. The additional bin was added to tabulate all the non- $u$ niform patterns.

Therefore, each histogram will be the feature vector that represents each image. Figure 2 and Figure 3 show four different retinal layers captured by OCTA scan for two eyes with different conditions, where the first figure shows a normal eye and the second figure shows an eye with wet AMD. In each figure, the texture of each retinal layer image was also measured with $\underline{r}=1$ and $p=8$ and this is to show how the $L B P s^{\text {riu } 2}$ values present the condition of wet $\mathrm{AMD}$ and normal of each particular layer. 

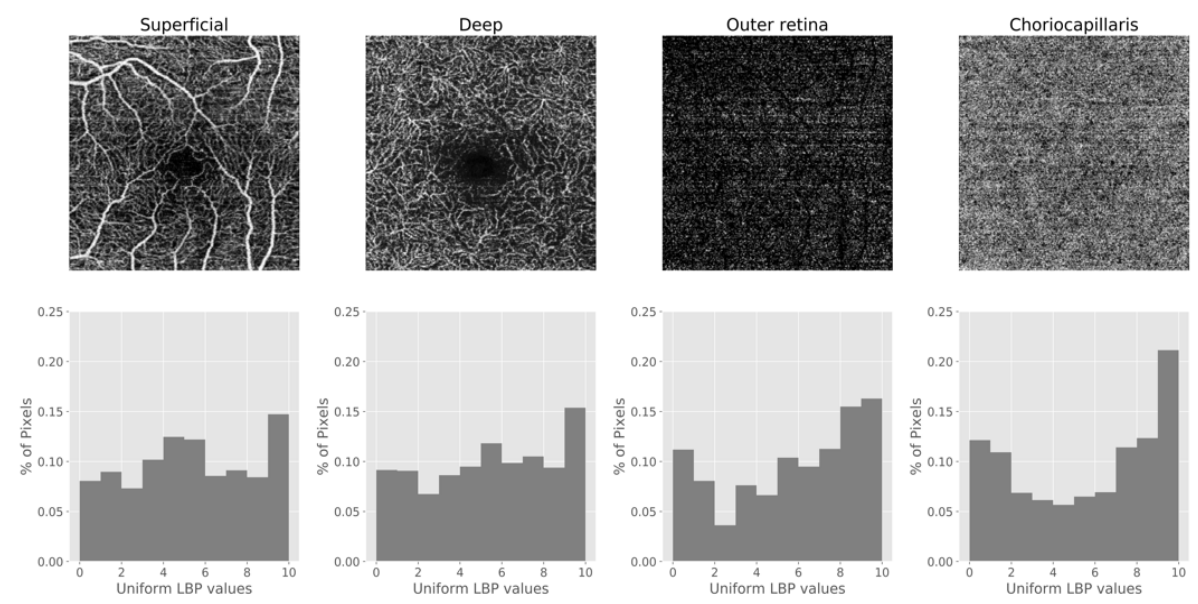

Fig. 2. The first row shows OCTA images for the superficial, deep, outer and choriocapillaris layers for a normal eye and the second row shows the histogram of $u$ niform $L B P s^{\text {riu } 2}$ values where $\%$ of Pixels refers to the percentage of pixels' number that share the same LBPs ${ }^{\text {riu } 2}$ values within each retinal layer image.
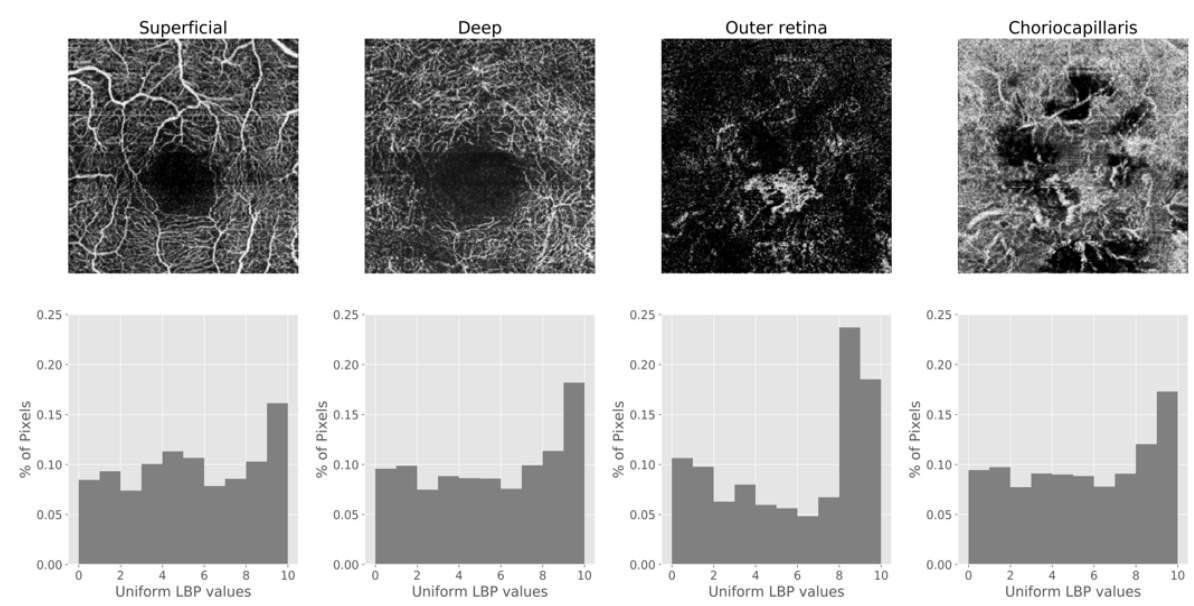

Fig. 3. The first row shows the OCTA images for the superficial, deep, outer and choriocapillaris layers for an eye with wet AMD and the second row shows the histogram of $u$ niform $L B P s^{r i u 2}$ values where \% of Pixels refers to the percentage of pixels' number that share the same $L B P S^{\text {riu2 }}$ values within each retinal layer image.

When the $L B P s^{r i u 2}$ values of both conditions are compared, it can be observed that the number of pixels that share the same pattern is almost comparable for the superficial and deep inner layers. However, it fluctuates in the outer and choriocapillaris layers due to the abnormalities in the capillaries caused by wet AMD. 


\subsection{Classification}

After obtaining these feature vectors (histograms), a classifier is then used to classify subsequent yet unseen OCTA images as having wet AMD or not. The classifier utilised in this study is K-Nearest Neighbour (K-NN). In the K-NN classifier, a data point is classified based on the majority vote of its $\mathrm{K}$ neighbours. In this study, the K$\mathrm{NN}$ classifier was utilised by empirically setting up the $\mathrm{K}=1$ neighbour. Choosing the $\mathrm{K}-\mathrm{NN}$ classifier to use in this study was motivated by the following:

1. Simple to implement, understand and interpret.

2. Requires no complex parameters tuning.

3. No learning stage involved.

4. Performs excellently with fairly well representative features.

5. Widely used to assess the performance of texture analysis methods, as in [10], [11] and [12].

\section{$4 \quad$ Evaluation and results}

The proposed algorithm was evaluated based on its performance in classifying OCTA images from various retinal layers as having wet AMD or normal control. These retinal layers are the superficial, deep, outer and choriocapillaris layers. Twenty-three normal control and twenty-three wet AMD OCTA images of each layer were used in this study, resulting in a total of 184 images. The images used in this study were provided by Manchester Royal Eye Hospital. The classification was performed on each separate retinal layer and on all layers together. The results were evaluated using different evaluation strategies and statistical measurements.

Validation sets were created using a ten-fold cross-validation strategy on all OCTA images. The mean accuracy (Acc) was estimated to assess the capacity of the algorithm to differentiate correctly between wet AMD and normal control cases. Moreover, the mean sensitivity (Se) was measured to assess the capacity of the algorithm to recognise the wet AMD cases and also the mean specificity (Sp) to assess its ability to recognise normal control cases. As a ten-fold cross-validation strategy was applied, the standard deviation of Acc, Se and Sp of all folds were measured. Extensive experiments were conducted to find the optimal combinations of $L B P s_{\underline{p}, \underline{r}}^{\text {riu }}$ parameters namely $\underline{r}$ and $\underline{p}$. For example results see Figure 4, 5 and 6. Table 1 summarises the classification results of the proposed algorithm showing the optimal combination of $\underline{r}$ and $\underline{p}$ for each classification experiment. 

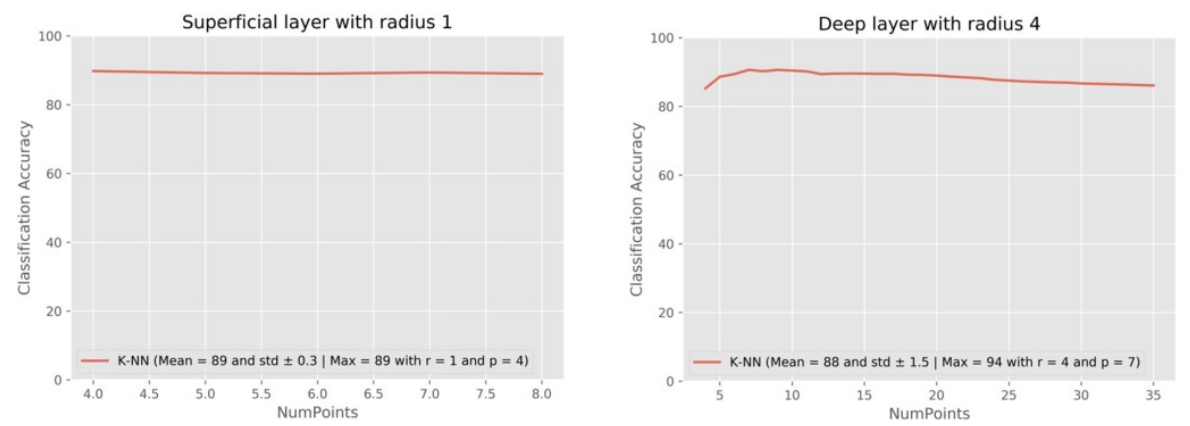

Fig. 4. Example results from the experiments conducted on the superficial and deep layers to find the optimal combination of $\underline{r}$ and $p$. Each line chart shows the mean classification accuracy results for the optimal radius with its possible number of points (NumPoints) with respect to each layer. The line charts that on the left is for the superficial layer and the one on the right for deep inner layer. The mean accuracy (Mean) and the standard deviation (std) are also presented when a different number of points around a particular radius was used as well as the maximum classification accuracy (Max) achieved in each experiment. The Max was achieved with $\underline{r}=1$ and $\underline{p}=4$ for the superficial layer and with $\underline{r}=4$ and $\underline{p}=7$ for the deep inner layer.
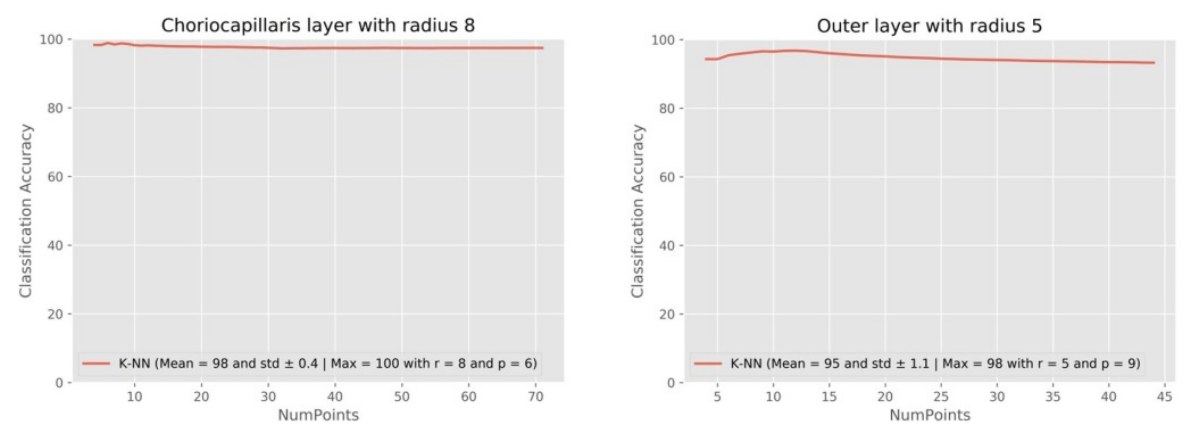

Fig. 5. Example results from the experiments conducted on the choriocapillaris and outer layers to find the optimal combination of $\underline{r}$ and $p$. Each line chart shows the mean classification accuracy results for the optimal radius with its possible number of points (NumPoints) with respect to each layer. The line charts that on the left is for the choriocapillaris layer and the one on the right for outer layer. The mean accuracy (Mean) and the standard deviation (std) are also presented when a different number of points around a particular radius was used as well as the maximum classification accuracy (Max) achieved in each experiment. The Max was achieved with $\underline{r}=8$ and $\underline{p}=6$ for the choriocapillaris layer and with $\underline{r}=5$ and $p=9$ for the outer layer. 


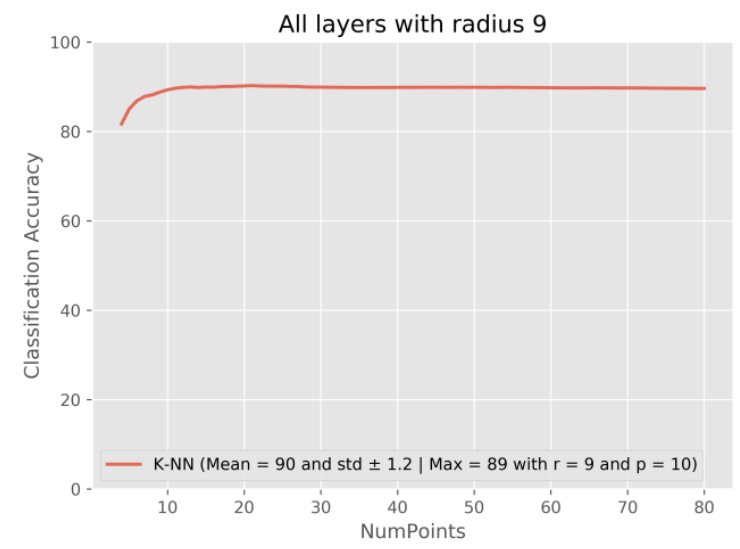

Fig. 6. Example results from the classification experiment conducted on all layers together using 92 normal control and 92 wet AMD images to find the optimal combination of $\underline{r}$ and $p$. This line chart shows the mean classification accuracy results for the optimal radius with its possible number of points (NumPoints). The mean accuracy (Mean) and the standard deviation (std) are also presented when a different number of points around a particular radius was used as well as the maximum classification accuracy (Max) achieved. The Max was achieved with $\underline{r}$ $=9$ and $p=10$

Table 1. Classification results of the proposed algorithm with the optimal combination of $\underline{r}$ and $p$ for processing each separate layer and all layers together.

\begin{tabular}{ccc}
\hline Retinal layers & Optimal LBPS $^{\text {riu }}$ parameters & K-NN results \\
\hline \multirow{3}{*}{ Choriocapillaris } & $\underline{r}=8$ & $\mathrm{Acc}=100 \% \pm 0.0$ \\
& $\underline{p}=6$ & $\mathrm{Se}=100 \% \pm 0.0$ \\
Outer & $\underline{r}=5$ & $\mathrm{Sp}=100 \% \pm 0.0$ \\
& $\underline{p}=9$ & $\mathrm{Acc}=98 \% \pm 5.3$ \\
& $\underline{r}=4$ & $\mathrm{Se}=100 \% \pm 0.0$ \\
Deep & $\underline{p}=7$ & $\mathrm{Sp}=96 \% \pm 10.7$ \\
& $\underline{r}=1$ & $\mathrm{Acc}=94 \% \pm 4.9$ \\
Superficial & $\underline{p}=4$ & $\mathrm{Se}=98 \% \pm 3.5$ \\
& & $\mathrm{Sp}=89 \% \pm 8.9$ \\
& $\underline{r}=9$ & $\mathrm{Acc}=89 \% \pm 8.9$ \\
All layers & $\underline{\mathrm{r}}=10$ & $\mathrm{Sp}=85 \% \pm 8.03$ \\
& & $\mathrm{Acc}=89 \% \pm 2.0$ \\
& & $\mathrm{Se}=93 \% \pm 2.2$ \\
& & $\mathrm{Sp}=86 \% \pm 2.0$ \\
\hline
\end{tabular}

From the evaluation results, it was observed that overall, the highest accuracy was achieved with the outer and choriocapillaris layers and this is due to the fact that the abnormalities in the blood vessels are clearer with these layers as compared to the 
other layers and hence these changes are reflected on the pixels of OCTA images. For example, in the choriocapillaris layer, the percentage of the pixels that share the same $L B P s^{r i u 2}$ patterns are constant with all OCTA images that constitute normal control. However, this is not the case with wet AMD images, where the percentage of the pixels that have the same $L B P s^{r i u 2}$ patterns has fluctuated due to the abnormalities in the capillaries caused by wet AMD.

The following figures illustrate the differences between wet AMD and normal control in OCTA images for the choriocapillaris layer. The texture of these images was measured by choosing $\underline{r}=8$ and $p=6$. Figure 4 shows normal control and Figure 5 demonstrates wet AMD. It can be seen clearly that the $L B P s^{\text {riu }}$ patterns with the normal control have smooth lines since the number of pixels that have the same $L B P s^{\text {riu } 2}$ patterns are constant; however, in the wet AMD they are not.

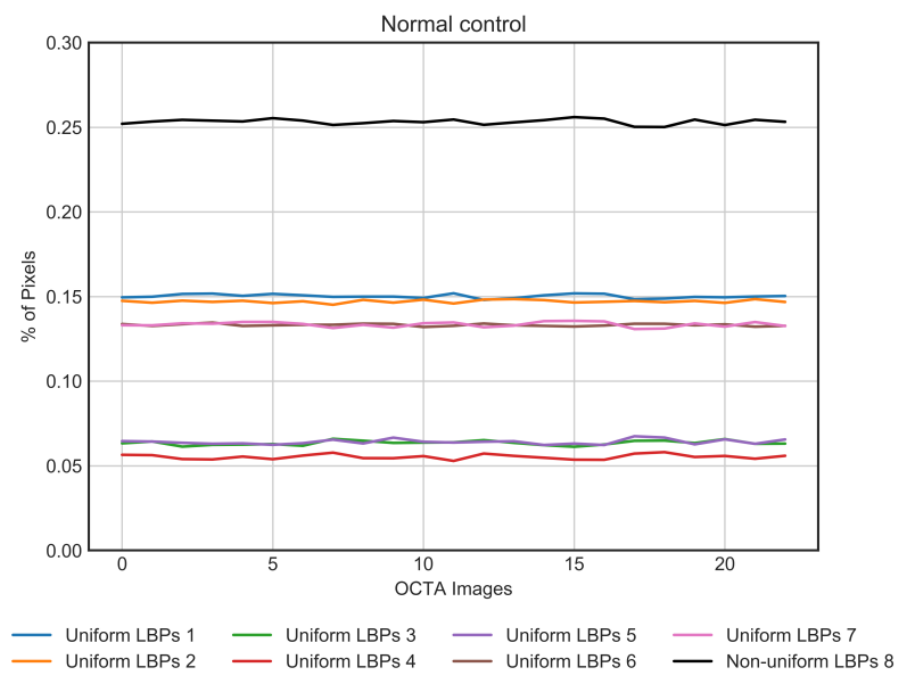

Fig. 7. The LBPs ${ }^{\text {riu2 }}$ patterns in OCTA images for the choriocapillaris layer with the normal control cases. The $\%$ of Pixels here refers to the percentage of the number of pixels that share the same $L B P s^{r i u 2}$ values within each image while OCTA images refer to the number of choriocapillaris layer images, which constitutes 23 images. 


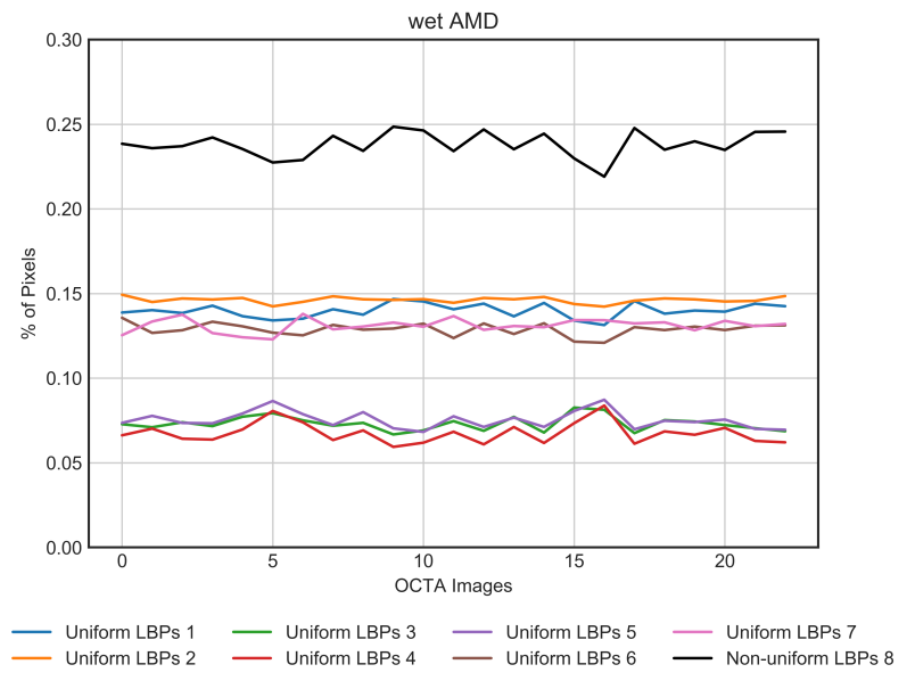

Fig. 8. The $L B P s^{r i u 2}$ patterns in the choriocapillaris layer with the wet AMD cases. The $\%$ of Pixels here refers to the percentage of the number of pixels that share the same $L B P S^{\text {riu2 }}$ values within each image while OCTA images refer to the number of choriocapillaris layer images, which constitutes 23 images.

\section{$5 \quad$ Discussion and conclusion}

This paper presents an age-related macular degeneration (AMD) classification algorithm based on local texture features to support the analysis of wet AMD in OCTA images. Due to the challenging nature of capturing images from patients' eyes using an OCTA scan, it can be difficult to ensure that all images have a similar rotation as well as to capture each one from the same field of view. To mitigate this problem, the algorithm employed the $L B P s^{r i u 2}$ descriptor that is invariant against texture orientation as well as any transformation of grey level change. With careful design, the algorithm does not need any manual assistance or segmentation for particular areas as it uses the entire OCTA image to obtain the features from. Then, it uses the features obtained to differentiate between the images that have wet AMD from those that constitute normal control. Accurate segmentation is hard to achieve when the characteristics of the diseases are complicated, hence incorrect diagnoses are more likely to occur with segmentation.

Four different retinal layers are tested in this study and the main purpose of using the various layers is to identify the layer that has the most discriminative information which describes the abnormal blood vessels patterns in wet AMD cases. Although the dataset used was relatively small, the algorithm achieved promising results in processing all layers together and each layer separately. The highest accuracies were achieved with the outer and choriocapillaris layers since the deformities of blood vessels on these layers are very clearly observable. The most important finding of this study is that employing the local texture features based on the LBPS ${ }^{\text {riuz }}$ descriptor in 
the classification using OCTA images achieved outstandingly accurate results. Future work involves and is not limited to the following: conducting extensive experiments to find the optimal value of $\mathrm{K}$ neighbours, evaluating its reliability for application in clinical ophthalmology, testing it on a large dataset to determine its strength and efficiency when a larger dataset is used and testing its capacity for identifying the severity of the disease by using a dataset that includes various stages of AMD cases.

\section{References}

[1] T. E. De Carlo et al., "Spectral-domain optical coherence tomography angiography of choroidal neovascularization," Ophthalmology, vol. 122, no. 6, pp. 1228-1238, 2015.

[2] Y. Jia et al., "Quantitative optical coherence tomography angiography of vascular abnormalities in the living human eye," Proc. Natl. Acad. Sci. U. S. A., vol. 112, no. 18, pp. E2395-402, 2015.

[3] T. E. de Carlo, A. Romano, N. K. Waheed, and J. S. Duker, "A review of optical coherence tomography angiography (OCTA)," Int. J. Retin. Vitr., vol. 1, no. 1, p. 5, 2015.

[4] L. Liu, S. S. Gao, S. T. Bailey, D. Huang, D. Li, and Y. Jia, "Automated choroidal neovascularization detection algorithm for optical coherence tomography angiography," Biomed. Opt. Express, vol. 6, no. 9, p. 3564, 2015.

[5] G. D. Tourassi, "Journey toward computer-aided diagnosis: role of image texture analysis," Radiology, pp. 317-320, 1999.

[6] T. Ojala, M. Pietikäinen, and T. Mäenpää, "Multiresolution gray-scale and rotation invariant texture classification with local binary patterns," IEEE Trans. Pattern Anal. Mach. Intell., vol. 24, no. 7, pp. 971-987, 2002.

[7] Y. Jia et al., "Quantitative optical coherence tomography angiography of choroidal neovascularization in age-related macular degeneration," Ophthalmology, vol. 121, no. 7, pp. 1435-1444, 2014.

[8] T. Ojala, M. Pietikainen, and D. Harwood, "Performance evaluation of texture measures with classification based on Kullback discrimination of distributions," in Pattern Recognition, 1994. Vol. 1-Conference A: Computer Vision \& Image Processing., Proceedings of the 12th IAPR International Conference on, 1994, vol. 1, pp. 582-585.

[9] R. F. Spaide, J. G. Fujimoto, and N. K. Waheed, Image artifacts in Optical Coherence Angiography, vol. 35, no. 11. 2015.

[10] J. Strand and T. Taxt, "Local frequency features for texture classification," vol. 27, no. 10, pp. 1397-1406, 1994.

[11] T. Ojala, M. Pietikäinen, and D. Harwood, "A comparative study of texture measures with classification based on featured distributions," Pattern Recognit., vol. 29, no. 1, pp. 51-59, 1996.

[12] P. P. Ohanian and R. C. Dubes, "Performance evaluation for four classes of textural features," Pattern Recognit., vol. 25, no. 8, pp. 819-833, 1992. 